\title{
Reporte de caso clínico: taquicardia supraventricular secundaria a irrigación con glicina
}

\author{
Case report: Supraventricular tachycardia secondary \\ to glicine irrigation
}

ELBA OROZCO GALVIS ${ }^{1}$, CATALINA MORENO-QUIJANO², SONIA MARGARITA VIVAS GARCÍA, MARÍA CAMILA GONZÁLEZ-ACEVEDO ${ }^{4}$

\begin{abstract}
Supraventricular tachycardia is one of the tachyarrhythmias that do not have its origin in one organic cardiomyopathy. Generally, they have a good prognosis, and its clinical spectrum varies from asymptomatic patients to those with low cardiac output. Treatment will depend on the hemodynamic stability of the patient. The objective of this case report is to make a review on existent literature about adverse cardiac effects with the use of Glycine in patients after urologic surgery.
\end{abstract}

\section{RESUMEN}

La taquicardia supraventricular hace parte del grupo de taquiarritmias que no se originan de una cardiopatía orgánica existente y por lo general son de buen pronóstico. Su espectro clínico abarca desde pacientes asintomáticos hasta aquellos con signos de bajo gasto cardíaco que requerirán tratamiento según su estabilidad hemodinámica. El objetivo del presente reporte de caso es realizar una revisión de la literatura sobre los efectos adversos asociados a la irrigación vesical continúa con glicina en un paciente a quien se le realizó prostatectomía

\section{Key words:}

Cystostomy, Glycine, Therapeutic Irrigation,

Tachycardia

Supraventricular, Complications.

\section{Palabras clave:}

Cistotomía, Glicina, Irrigación Terapéutica, Taquicardia

Supraventricular, Complicaciones.

Médica anestesióloga, Universidad Industrial de Santander, profesora asistente de Anestesiología y Reanimación Universidad Industrial de Santander, Hospital Universitario de Santander, Bucaramanga, Colombia.

2 Residente de Anestesiología y Reanimación, segundo año, Universidad Industrial de Santander, Bucaramanga, Colombia. 3 Médica general de Unidad de Cuidados Posanestésicos, Hospital Universitario de Santander, Bucaramanga, Colombia.

4 Médica general, Uromédica, Clínica Chicamocha, Bucaramanga, Colombia.

Fecha de recepción: 18 de febrero de 2018

Fecha de aceptación: 15 de abril de 2018

\section{ORCID}

orcid.org/0000-0002-3745-8344

Correspondencia:

Sonia Margarita Vivas García

soniamargaritavivasgarcia@gmail.com 
abierta más cistostomía suprapúbica como tratamiento para hiperplasia prostática benigna refractaria a manejo médico con alfa bloqueadores uroselectivos. Caso clínico de paciente masculino de 81 años sometido a prostatectomía abierta más cistostomía suprapúbica por el servicio de urología como tratamiento para hiperplasia prostática benigna (HPB) refractaria a manejo médico. Se realiza anestesia regional habitual y se indica irrigación vesical continua en el postoperatorio con glicina al 1,5\%. Luego de 8 horas de postoperatorio el paciente presenta taquicardia supraventricular atribuible a la irrigación por glicina y es manejado con amiodarona con buena respuesta y resolución de la taquiarritmia. Se realiza discusión sobre el uso de glicina en irrigación vesical y las complicaciones asociadas al uso de la misma.

\section{Introducción}

a taquicardia supraventricular (TV) es un trastorno del ritmo cardíaco que engloba una serie de ritmos rápidos y regulares, que se dan por encima de la bifurcación del haz de His[1,2]. La más común es la reentrada en el nodo auriculo ventricular (nodo AV), que corresponde al $60 \%$ de los casos y es una de las formas de taquicardia con complejo QRS estrecho. Clínicamente se caracteriza por ser de buen pronóstico y en la mayoría de los casos no se encuentra una cardiopatía orgánica preexistente[2-3].

El espectro clínico de la TV varía desde pacientes asintomáticos hasta aquellos que manifiestan signos de bajo gasto cardíaco y episodios sincopales, 2 lo cual determinará el tratamiento teniendo en cuenta la estabilidad hemodinámica del paciente; aquellos con TV inestable requieren de cardioversión eléctrica inmediata y aquellos con TV estable serán candidatos a maniobras vagales y antiarrítmicos endovenosos como la adenosina y la amiodarona[3].

\section{Caso clínico}

Adulto mayor de 81 años, masculino, sin registro de antecedentes patológicos o farmacológicos de importancia confirmado durante la valoración preanestésica y revisión de historia clínica previa. En la revisión de paraclínicos prequirúrgicos se evidencia radiografía de tórax con prominencia del botón aórtico y escoliosis torácica, electrocardiograma con ectopia ventricular aislada, sin signos de isquemia; ecografía pélvica: Próstata de aproximadamente 115 cc, no se cuenta con electrolitos prequirúrgicos. El anestesiólogo que realiza la valoración lo considera categoría 2 según la clasificación de estado físico del ASA 4; No se conocen datos adicionales del preparatorio del paciente.
Programado para prostatectomía abierta más cistostomía suprapúbica por diagnóstico de hiperplasia prostática benigna (HPB) por el servicio de urología.

Se realiza cirugía bajo anestesia subaracnoidea con bupivacaina 12,5 mg única punción a nivel de L3 lográndose nivel sensitivo T6. Durante el intraoperatorio permanece hemodinámicamente estable, sin requerimiento de soporte vasopresor. Tiempo quirúrgico de 3 h, líquidos endovenosos administrados 2.250 cc de lactato de Ringer y 9.000 cc de cistoirrigacíon con glicina al 1,5\%, sangrado aproximado de 700 cc por lo que se inició manejo con ácido tranexámico $1 \mathrm{~g}$ endovenoso. (anexo 1: registro de anestesia).

Tras $8 \mathrm{~h}$ del post operatorio y bajo vigilancia en unidad de cuidados posanestésicos el paciente presenta frecuencia cardíaca de 202 lat/min, ritmo de taquicardia en cardiovisoscopio en derivación DII, presión arterial de 104/87 mmHg, oximetría de pulso de $97 \%$. Al interrogatorio no refiere ningún síntoma asociado, niega dolor precordial, disnea, cefalea y mareo. Se realiza electrocardiograma que evidencia ausencia de onda $\mathrm{P}$, confirmado en diferentes velocidades por el equipo de EKG, complejos QRS regulares y angostos, frecuencia cardíaca de 250 lat/min compatible con taquiarritmia. No se identifican signos de isquemia o necrosis. (anexo 2: ekg).

Basados en los hallazgos clínicos y electrocardiográficos se diagnostica taquicardia supraventricular con estabilidad hemodinámica por lo cual se inicia manejo según algoritmo terapéutico 5; se inicia masaje carotídeo durante 10 segundos previa auscultación de vasos carotídeos durante 2 oportunidades sin éxito, por lo cual se indica aplicación de adenosina endovenosa que no se encontraba disponible en el hospital. Continuando la secuencia terapéutica se decide cardiovertir farmacológicamente con amiodarona $150 \mathrm{mg}$ en $15 \mathrm{~min}$ la cual logró retorno a ritmo sinusal con éxito, logrando frecuencia cardíaca de 90 


\begin{tabular}{|c|c|c|}
\hline & Inicial & Control \\
\hline Hemoglobina & 10 & 11 \\
\hline Hematocrito & 30 & 34 \\
\hline Troponina I & 1,50 & 3,50 \\
\hline Creatinquinasa & 117 & \\
\hline$C K-M B$ & 13,20 & \\
\hline & $\begin{array}{l}\mathrm{PH} 7,43 \\
\mathrm{PCO}_{2} 36 \\
\mathrm{PO} 2115\end{array}$ & \\
\hline Gases Arteriales & $\begin{array}{c}\mathrm{HCO} 323 \\
\mathrm{BE}-0,5 \\
\mathrm{CA} 99 \\
\mathrm{~K} 4,33 \\
\mathrm{CL} 4,39 \\
\mathrm{NA} 137\end{array}$ & \\
\hline Electrolítos & $\begin{array}{c}\text { NA } 140 \\
\text { K } 4,7 \\
\text { CL } 104\end{array}$ & $\begin{array}{l}\text { NA } 137 \\
\text { K } 3,97 \\
\text { CL } 100\end{array}$ \\
\hline
\end{tabular}

latidos/minuto sin signos de isquemia o necrosis en electrocardiograma control. Se realizó seguimiento con laboratorios reportados en Tabla 1. (anexo 3: tabla de paraclínicos)

Se consideró requería valoración por medicina interna y cardiología para descartar posibles etiologías de taquiarritmia y monitoreo en unidad de cuidado intermedio, sin embargo, en la institución no se cuenta con dicha unidad y no había disponibilidad de cubículo en unidad de cuidados intensivos.

El servicio de medicina interna considera cambios electrocardiográficos no asociados a trastorno del equilibrio ácido base, ni electrolítico o de la oxigenación. Fue valorado por cardiología quien realizó estudios de extensión: Ecocardiograma con FEVI 62\%, insuficiencia aórtica leve y calcificaciones de válvulas sigmoideas, adicionalmente biomarcadores negativos. Dado que no se encontró ninguna causa desencadenante del evento cardíaco se asoció a la irrigación vesical con glicina.

Se revisa registro de control de irrigación urinaria encontrando 30.000 cc de glicina al 1,5\% (45 g de glicina) irrigada por lo que se indica disminuir velocidad de administración de la misma.

Teniendo en cuenta lo anterior se considera cuadro de efecto adverso por medicamentos, específicamente asociado al uso de glicina por lo que servicio tratante urología cambia a irrigación con Lactato de Ringer.

Durante la vigilancia el paciente continua hemodinámicamente estable, sin nuevos cambios en ritmo cardíaco, por lo que se considera episodio de taquiarritmia supraventricular paroxística, sin antecedentes cardiovasculares ni patología coronaria, quien puede continuar manejo y seguimiento en sala de hospitalización general a cargo del servicio de urología; no se tienen datos adicionales de nuevos episodios de taquiarritmia durante la estancia hospitalaria y egresa sin ninguna complicación adicional.

\section{Discusión}

La hiperplasia prostática benigna (HPB) es el aumento de tamaño de la glándula en ausencia de cáncer[6]. Dentro de los factores de riesgo más importantes se encuentran la edad, testículos funcionales, raza (negra), historia familiar de la patología y factores dietarios específicamente consumo de vegetales con alto contenido de fitoestrógenos, que tienen efectos antiandrogénicos sobre la próstata[7]. La prevalencia de HPB aumenta de forma directamente proporcional a la edad, observándose en un 50\% de los hombres de 60 años y en un $90 \%$ a los 85 años[6].

En el manejo terapéutico de esta patología, se destacan las técnicas quirúrgicas claramente superiores a las otras formas de tratamiento, en cuanto a la mejoría de síntomas considerándose hasta la fecha como el "patrón de oro" para el manejo de la misma[6]. El tamaño de la glándula y la severidad de los síntomas determinará el tipo de intervención quirúrgica a realizar[7].

La resección transuretral de la prostáta (RTUP) ofrece mejoría sintomática del $90 \%$ y se indica para glándulas menores de $60 \mathrm{ml}$. La prostatectomía abierta se indica para próstatas mayores de $60-70 \mathrm{ml}$ y la incisión transuretral útil para próstatas de tamaño pequeño (< de $30 \mathrm{ml}$ )[7]. En este paciente se escogió la técnica abierta por el volumen prostático reportado en la ecográficamente.

La glicina al 1,5\% en agua destilada es la solución hipotónica más utilizada para cistoirrigación, por ser un soluto hidrosoluble, no electrolítico, poco hemolítico, con una osmolaridad es de 200 mosml y sin metabolismos tóxicos[8]. La concentración desencadenante de toxicidad por glicina suele ser mayor a los $800 \mathrm{mmol}$, es por esto que durante la intervención quirúrgica los signos clínicos no dependen directamente de los niveles de glicina, si no de la hipervolemia y la capacidad del individuo para metabolizarla. El paciente no presentó ningún síntoma ni signo clínico en el intraoperatorio.

Los signos iniciales de hiperglicinemia incluyen nauseas, vomito, cefalea, y debilidad muscular, que 
se desencadenan después de la absorción de 22-35 g de glicina que equivalen a aproximadamente 15.000 35.000 litros de glicina al 1,5\%; por lo tanto, el mejor índice del aumento de glicina en plasma es la disminución de la concentración de sodio[9]. El paciente descrito recibió irrigación de 30.000 cc lo que equivale a $45 \mathrm{~g}$ de glicina en 8 horas.

La glicina tiende a distribuirse a todos los compartimentos hídricos del organismo, dejando el agua libre en el plasma, lo cual produce hiposmolaridad plasmática que asociada a la hipertensión e hiponatremia causan desplazamiento del agua del sector extracelular hacia el intracelular ocasionando una disminución de la presión venosa central, hipotensión y edema; se cree que el bloqueo simpático inducido por la anestesia regional puede exacerbar estos signos clínicos, así como el sangrado y la hemólisis $[8,10]$.

Teniendo en cuenta lo anterior se ha demostrado que la glicina puede ser tóxica para el sistema cardiovascular y sistema nervioso central si se infunde en grandes cantidades, produciendo cambios en el ecocardiograma y aumento de troponina I[11]. Dentro de su efecto tóxico miocárdico bifásico se desarrolla primero un estado hiperdinámico con las resistencias vasculares periféricas reducidas, seguidas de una fase de bajo gasto que activa el sistema beta-adrenérgico explicando estos posibles cambios[9].

En varios estudios que han evaluado los efectos de la glicina en el corazón en animales (ratones) han encontrado edema del miocardio, lo que genera bradicardia y prolongación de la duración del QRS en el electrocardiograma, que pueden observarse incluso entre 2 y 7 semanas después de haber recibido la solución de glicina[12]. En humanos, se observó la liberación de enzimas cardíacas y aplanamiento subagudo de la onda T en pacientes sometidos a cirugía de próstata que absorbieron de 1 a 3,5 litros del líquido, lo cual relaciona la glicina con cambios isquémicos en el electrocardiograma a diferencia de otras soluciones hipotónicas también utilizadas para cistoirrigacion[12].

Los síntomas neurológicos de la hiponatremia se relacionan con la severidad y el tiempo de instauración en la disminución de los niveles de sodio; estos son debidos al edema cerebral, producto del movimiento de agua desde el LEC al interior de las neuronas, ocasionando un incremento de la presión intracraneana; así mismo conforme los niveles de sodio disminuyen se hace inminente la herniación del tronco cerebral, provocando paro respiratorio, coma y muerte[13].

El cuadro clínico caracterizado por hipervolemia, hiponatremia y toxicidad del líquido de irrigación, se conoce como el síndrome de resección transuretral de la próstata, que tiene alto riesgo de manifestaciones cardiovasculares y neurológicas por lo cual requiere de monitoría invasiva intraoperatoria y dirigir el tratamiento hacia soporte cardiovascular, inotropía, expansores de volumen plasmático, administración de calcio, corrección de hiponatremia y medidas encaminadas a disminuir el riesgo de edema cerebral tales como la hiperventilación[14]; así mismo se debe tener en cuenta las mediciones séricas de electrolitos, seguimiento electrocardiográfico, hemático y gases arteriales cuando se sospeche intoxicación por líquidos de cistoirrigación; adicional la administración de L-arginina endovenosa también se ha considerado útil en el tratamiento de la hiperamoniemia asociada al metabolismo de la glicina, sin embargo, ante la duda clínica se debe tener en cuenta otras alternativas en la composición de la irrigación como es el lactato Ringer, sorbitol al $2,5-3 \%$ y manitol al $0,54 \%$ pero también hay que tener en cuenta su hipotonicidad semejante a la glicina al 1,5\%[14].

Teniendo en cuenta lo anterior y el cuadro clínico descrito consideramos que el paciente presento una intoxicación por glicina, correlacionando la clínica con la cantidad de la misma administrada. Por otra parte, no se contaba con medición de sodio basal para realizar un comparativo con el que se presentó durante y posterior el evento para confirmar la hiponatremia secundaria a hipervolemia que presentan en este caso. Adicionalmente vale la pena resaltar el inconveniente con la no disponibilidad de adenosina, fármaco de primera línea en el algoritmo de manejo, ni cubículo en unidad de cuidados intermedios para una monitoría y seguimiento, razón por la cual ésta se realiza en salas de hospitalización general.

\section{Referencias}

1. Gándara J, Santander D, Mora $\mathrm{G}$, et al, Taquicardias supraventriculares. estado del arte, Rev. Fac. Med. 2016; 64 (1) 111- 21.
2. Almendral J, Castellanos E, Ortiz M, Taquicardias paroxísticas supraventriculares y síndromes de preexcitación, Rev Esp Cardiol. 2012; 65:456-69.

3. Galduf J, Monte E, Escrivá JJ, et al, Adenosina y derivados en el tratamiento de las taquicardias supraventriculares. Farm Hosp 1995; 20 (5): 279-288.

4. ASA Physical Status Classification System [Internet]. American So- 
ciety of Anesthesiologists, 2014 [ citado 4 de abril de 2018] disponible en: https://www. asahq.org/resources/clinicalinformation/asa-physical-statusclassification-system.

5. Gandara J, Santander D, Taquicardias Supraventriculares: Estado del Arte. [Tesis doctoral en Internet]. Bogotá. Universidad nacional de Colombia, 2014, [citado 10 de noviembre de 2017] disponible en: http://www.bdigital.unal.edu. co/12855/1/5599166.2014.pdf

6. Gómez P, López H, Moreno M, et al, Guía de Practica Clínica Hiperplasia Prostática Benigna Diagnóstico y Tratamiento [Internet]. Sociedad Colombiana de Urología. 2014. [ citado 10 de noviembre de 2017]. Disponible en: http://scu.org.co/userfiles/file/ guias2015/4\%20guia\%20de\%20 manejo\%20hiperplasia\%20prostatica\%20benigna.pdf

7. Sierra M, Catalá L, Monedero L, Hiperplasia Benigna de Próstata, Guía de Actuación Clínica en Atencion Primaria [Internet]. Hospital clínico Valencia. Disponible en: http://www.san.gva. es/documents/246911/251004/ guiasap020prostata.pdf

8. Metje MT. Anestesia en Cirugía Urológica y en la Insuficiencia Renal, disponible en: http:// www.scartd.org/arxius/urologia05.pdf

9. Caba F, Campos JM, Herrera A, et al. Complicaciones durante ka anestesia. En: Torres L.M, director. Tratado de Anesthesia y Reanimacion. Vol 2, España: Aran; 2001. Pág 2044.

10. Senthilkumar $V$, Turp Syndrome. Trends in Anaesthesia and Critical Care. (2011)1 (1): 46-50.

11. Ayman $Y$, Ghada S, Osama E, et al, A randomized comparison between three types of irrigating fluids during transurethral resection in benign prostatic hiperplasia; BMC Anesthesiology 2010, 10:7.

12. Hahn $R$, Glycine $1.5 \%$ for Irrigation Should be Abandoned, Urol Int 2013; 91:249-255 12.

13. Castellanos L, Cardenas L, Carrillo M, Revisión de hiponatremia, Horiz. Med 2016; 16 (4).

14. Forero J, Guzmán C. Síndrome de resección trasuretral de próstata, rev, col, urol1996; 2 (03). 